\title{
UNA INSCRIPCIÓN JONIA ARCAICA EN HUELVA
}

\author{
JESÚS FERNÁNDEZ JURADO \\ Museo Arqueológico de Huelva \\ RICARDO OLMOS \\ Museo Arqueológico Nacional
}

\begin{abstract}
Se estudia en este artículo un fragmento de cuenco, probablemente milesio, que procede de las excavaciones de la calle del Puerto, en el establecimiento tartésico de Huelva. En el exterior del labio existe una inscripción jónica arcaica, de mediados del siglo VI a.C., cuya interpretación es particularmente difícil. Puede ser una dedicación u ofrenda hecha a un jefe local por un griego, pero nada puede afirmarse con total seguridad.
\end{abstract}

We analyse in this paper a bowl fragment, probably milesian, which comes from the excavations of Calle del Puerto, in the tartessian settlement of Huelva. Along the outside lip runs an archaic ionian inscription -middle of the 6th c. B.C.- whose interpretation is particularly difficult. It may be a dedication or offering made by a Greek for a local chief. But nothing can be said about it with certainty.

El alto interés arqueólogico que la ciudad de Huelva ha tenido para los investigadores se ha visto incrementado en los últimos años, gracias a los hallazgos que se han ido produciendo en el casco antiguo de la ciudad (OLMOS y CABRERA, 1980, 5-14; OLMOS y GARRIDO, 1982, 243-259; FERNÁNDEZ-JURADO, 1984; ídem, 1985; CABRERA y OLMOS, 1985).

Ante esta importancia y dado el evidente peligro de desaparición de los restos arqueológicos existentes en el subsuelo de la ciudad, como consecuencia del rápido ritmo de las construcciones de nueva planta, el Servicio de Arqueología de la Diputación Provincial de Huelva ha desarrollado, desde 1982, un amplio plan de excavaciones (fig. 1).

Los trabajos realizados hasta el momento han proporcionado abundantes muestras de cerámicas arcaicas griegas, destacando en cuanto a volumen de piezas, calidad de las mismas y talleres de procedencia, las excavaciones efectuadas en la calle Méndez Núñez, solares 4-6 y 5, así como en Puerto, 9, lugar donde fue hallada la pieza objeto de este estudio.

\section{LA EXCAVACIÓN}

El solar no pudo ser excavado en su totalidad, ya que se había realizado el pilotaje necesario para la cimentación del nuevo edificio; no obstante, en la zona sur del mismo, pudimos aprovechar el espacio destinado a patio de luces, lo que nos permitió trabajar sobre una cuadrícula de $6,50 \times 3,80 \mathrm{~m}$.

Dadas las características de urgencia de la excavación, ésta se realizó mediante el levantamiento de planos artificiales, en la mayoría de las ocasiones, $y$ que no sobrepasaron en ningún momento $\operatorname{los} 12 \mathrm{~cm}$. de espesor. La máxima profundidad alcanzada ha sido $-5,73 \mathrm{~m}$., en la que hubo que 


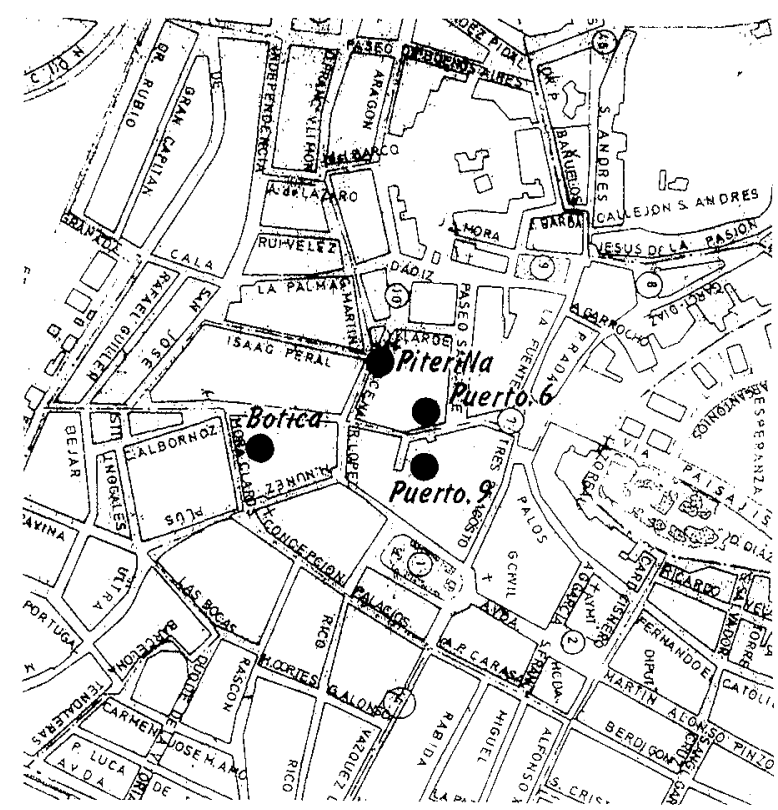

Fig. 1

abandonar los trabajos por la presencia de agua. En realidad y desde la cota de $-4 \mathrm{~m}$., se había tenido que bombear el agua que iba apareciendo y que dificultaba la excavación, lo que unido al peligro de derrumbes y a la imposibilidad de continuar con las mínimas garantías científicas, nos obligó a no proseguir los trabajos.

Ante la evidencia expresada y sabiendo que la estratigrafía arqueológica tenía aún mayor potencia, procedimos a realizar un sondeo mecánico con barrena de $15 \mathrm{~cm}$. de diámetro, que nos diera idea de la amplitud total de la estratigrafía que quedaba sin excavar. El sondeo puso de manifiesto que, en ese punto de la ciudad, la estratigrafía arqueológica alcanza los $8 \mathrm{~m}$. de potencia total.

\section{LA CERÁMICA}

La pieza que estudiamos presenta un doble interés: de una parte, la funcionalidad de esta cerámica; de otra, la inscripción realizada sobre ella (CARRIAZO y RADDATZ, 1960, 103-104, estrato III; PELLICER y AMORES, 1985, 57-189, estrato VI).

Realizada a torno, presenta un diámetro de 25 $\mathrm{cm}$. y un espesor de $7 \mathrm{~mm}$., poseyendo un borde aristado y ligeramente engrosado al interior. La pasta, bien depurada, es de tono amarillento, siendo los desgrasantes finos, con abundancia de mica y numerosos puntos de cal. La cocción oxidante es uniforme y de buena calidad.

Las superficies aparecen cuidadas y bien alisadas, con fino engobe del mismo color de la pasta, teniendo mejor tratamiento el exterior que el interior y apreciándose dos finas incisiones paralelas a aquélla.

A primera vista y si no se hace un análisis detallado del fragmento cerámico, se consideraría que el mismo es una producción local enmarcable en el contexto de los cuencos oxidantes de tradición fenicia. Ubicarlo en este grupo sería fácil y se encontrarían posibles paralelos del mismo en Carmona (LUZÓN y RUIZ MATA, 1973, estrato 10), la Colina de los Quemados (PELLICER y otros, 1983, nivel 21), el cerro Macareno (ARRIBAS y WILKINS, 1971) o la necrópolis de Frigiliana (VOITGLANDER, 1982, lám. 40, n. 252-7), por sólo citar algunos de los numerosos yacimientos donde pueden hallarse.

Sin embargo, la factura de la pieza, su pasta y desgrasantes, entre otras consideraciones, hacen difícil integrarla en el grupo de las producciones locales, máxime teniendo en cuenta el hecho de la inscripción misma realizada en griego. Por otra parte, sería aventurado afirmar categóricamente que se trata de una producción local, cuando cuencos de este tipo aparecen en ambientes griegos, como puede ser el caso de Mileto (VOITGLANDER, 1982), aunque en algunas ocasiones usados como tapaderas, que también pudiera haber sido el caso de esta pieza de Huelva.

En realidad, es sumamente difícil definirse por una funcionalidad u otra, teniéndose que considerar además el hecho de que las cerámicas de este tipo pueden ser usadas indistintamente como cuencos o tapaderas.

Una primera impresión hace pensar en su uso como tapadera, dado el mejor tratamiento de la superficie externa. Igualmente, podría afirmarse que éste fue su uso dado el lugar donde se realizó la inscripción, facilitando así su lectura; sin embargo y como más adelante se verá, no es extraño en el mundo griego que las inscripciones se realicen junto al borde de las piezas. Sea como fuere, es decir, se usara como cuenco o tapadera, no es ésta la finalidad de nuestro escrito, ni tampoco radica en ello la importancia de la pieza, sino en la inscripción que se efectuó sobre la misma.

En el nivel estratigráfico en que fue hallada la pieza, fechable en la primera mitad del siglo VI a. 
de C., predominan las cerámicas realizadas a torno, es significativo aún el número de las fabricadas a mano y son escasas las de técnica bruñida, llamando la atención el elevado porcentaje que representan las cerámicas griegas (Cuadro I), algo frecuente en esta y otras excavaciones que hemos realizado en Huelva, lo que pone de manifiesto la importancia de la presencia del mundo griego arcaico en el suroeste peninsular.

\section{CUADRO I}

Cerámicas a torno

Cerámicas a mano

Cerámicas bruñidas

Cerámicas griegas
2.423 fragmentos

323 fragmentos

5 fragmentos

83 fragmentos
$85,50 \%$

$11,40 \%$

$0,18 \%$

$2,92 \%$

\section{J. F. J.}

\section{LECTURA E INTERPRETACIÓN DE LA INSCRIPCIÓN (*)}

Un problema previo que plantea la inscripción del plato de Huelva es la lectura de sus signos debido al deterioro material de la superficie.

La capa más externa de la cerámica, una fina película con el alisado final, ha desaparecido parcialmente y con ella algunos trazos, generalmente los menos penetrantes, de las letras. Está especialmente dañada la parte izquierda del fragmento, en una zona que ocupa, aproximadamente, las dos primeras letras perdidas y que indicamos en el dibujo con un rayado. Nos vemos, pues, abocados a conjeturar más que a definir. Se une a ello la existencia en este lugar de rasguños accidentales que fácilmente podrían interpretarse, en un primer momento, como trazos de las letras perdidas lo que aumentaría la confusión interpretativa.

Hoy leemos:

vNInOM:

siendo ya dudoso el minúsculo trazo izquierdo de la primera letra, la supuesta N. Ello nos lleva a la siguiente lectura:

\section{].vฺıı $\delta \omega !$}

Se trata, sin duda, de una inscripción jonia arcaica. Es característica del jonio la introducción de la $H$ y $\Omega$ para distinguir la coloración larga y abierta de la $e$ y $o$ frente a las respectivas e y o (breves y cerradas), cuya notación es E y O (JEFFERY, 1961, 325-7; GUARDUCCI, 1967, 259 ss.; WOODHEAD, 1981, $16 \mathrm{ss}$.). La theta con la cruz inscrita es normal en los alfabetos arcaicos hasta que paulatinamente la cruz se sustituye por un punto: $\odot$ (1). La $N$ inclinada y con y trazo izquierdo más largo y bajo que el derecho es asimismo normal en el siglo VI. La $\Omega$ con el trazo izquierdo prácticamente vertical, denota la característica irregularidad de la escritura jonia (2), como señala en su caracterización general a la misma, Miss Jeffery: «It differs from the contemporary script of the rest of Greece, being smaller and more carelessly formed. This suggests that the Ionians were familiar at an earlier period than the other Greeks with the practice of writing on leather rolls》 (JEFFERY, 1961, 327).

En nuestro fragmento pueden observarse estas características en la disposición descuidada de las letras (por ej., la $\mathrm{H}$ ), o en la misma espontaneidad en el tratamiento del espacio: no constriñe la secuencia gráfica en un rígido marco o pauta horizontal. Por el contrario, las letras se mueven libremente, aunque guardan un interespacio homogéneo entre ellas, lo que sí indica un cuidado intencional. Es ésta, en cierto modo, una escritura precursiva, lo que va a ser general en los grafitos griegos.

Dentro del mundo jonio arcaico resulta ya difícil precisar más la procedencia concreta de la inscripción. Podría ser indistintamente, en nuestro caso, samia o focea. La forma abierta de la $\mathrm{H}$
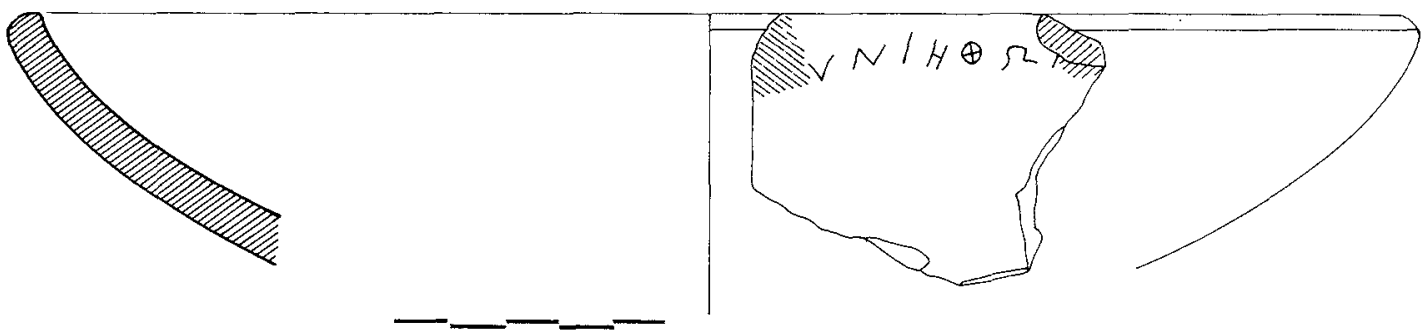

Fig. 2 
(frente a la grafía más arcaica $\boxminus$ ) se documenta ya en Quíos a finales del siglo VII a. C. (GUARDUCCI, 1967, 259); en Samos está ya en uso hacia el 570 a. C., y en Éfeso, según Jeffery, hacia mediados del siglo (3). Arqueológica (por el contexto) y epigráficamente nuestra inscripción encaja aproximadamente en esta fecha, la primera mitad del siglo VI a. C., tal vez el segundo cuarto del siglo VI a. C., coincidiendo, pues, con el esplendor del comercio griego en Tarteso.

La situación de la inscripción junto al borde exterior del vaso es normal, por ejemplo, en las copas de labio jonias (T $\Omega \Pi \Omega \Lambda \Lambda \Omega N$ : A Apolo (4); HPHI: A Hera (5), o en el famoso rhytón en bronce, rematado en cabeza de toro, ofrecido por Diágoras a la Hera de Samos (KYRIELEIS, 1981, 20 fig. 8; HOMANN-WEDE-KING， 1966，554; KOPCKE, 1968, 289-90, lám. 121, 1, 2). En estos ejemplos, que son dedicaciones de ofrendas a los dioses, ocupa aquélla un lugar relevante del vaso, es decir, allí donde resulta más inmediata su lectura (6). En estos ejemplos el carácter irregular, precursivo, de las inscripciones es notable.

La interpretación del fragmento es difícil y, hoy por hoy, no debe dejar de ser conjetural. Nuestra propuesta parte de que, efectivamente, la primera letra conservada sea una $\mathrm{N}$ ( $\mathrm{y}$ no una $\mathrm{V}$, que sería la otra alternativa teóricamente posible),

(*) Quiero expresar aquí mi agradecimiento a Miss Jeffery por su amabilidad y sugestiva in formación al discutir personalmente con ella en Oxford (nov. 84) este fragmento. Agradezco asimismo al Prof. J. de Hoz (Salamanca) sus valiosas observaciones a mi manuscrito, especialmente la referencia de Cristofani, 1975.

(1) Guarducci, 1967, 259: «la variante la più recente e già presente nelle iscrizione vasculari, verso la metà del VI secolo».

(2) Guarducci, 1967, 260: «il típo più antico presenta la sola apendice de destra».

(3) JEFFERY, 1961, 325: Miss Jeffery, sin embargo, moderniza en varias décadas la fecha. Para una mayor antigüedad de la $\mathrm{H}$ abierta, ef. GUARDUCCI, 1964, 133.

(4) Museo Británico, $n^{\circ} 86 / 4-1 / 309$, de Naúcratis. PETRIE, 1886, lám. XXXII, 162.

(5) Museo Británico. Inédita. $N^{\circ}$ 1965/9-30/1971, de Naúcratis. Del estudio actual de estas inscripciones vasculares arcaicas del Museo Británico se ocupa A. W. Johnston, a quien agradecemos su amable información. En una y otra copa la escritura es muy irregular. Cf. asimismo WILLIAMS, 1983, 169 78 , inscripciones pintadas junto al labio en los cántaros quiotas de Egina. lo que conjeturamos en base al breve trazo izquierdo que puede ya hoy distinguirse con cierta dificul$\operatorname{tad}(7)$.

De aceptar eșta letra $\mathrm{N}$ lo más verosímil es que tengamos entonces que dividir la secuencia inmediatamente después de ella, esto es, proponer aquí el final e inicio, respectivamente, de dos palabras distintas, pues, en caso contrario, la escritura arcaica jonia habría notado preferentemente con una consonante simple la geminada. Conocemos pocas excepciones a esta regla, por ejemplo la estela boustrophédon de Sigeion, conservada en el Museo Británico, donde se escribe ПPOKONNH乏IO (8).

Seguramente incluso en este caso existe una conciencia lingüística del carácter compuesto del topónimo, cuyo segundo término es nesos.

Aceptada la división de palabras (sin signos de interpunción como es normal, por lo que hemos podido ver, en las dedicaciones jonias de las copas arcaicas) y aceptado también el hecho de que debamos leer este fragmento de izquierda a derecha (pues la lectura retrógrada carece totalmente de sentido), proponemos conjeturalmente la interpretación:

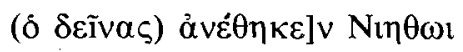

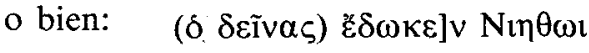

«... me dedicó ( me dio) a Nieto»

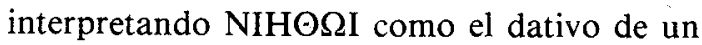
nombre

(6) Existen también numerosos ejemplos en que la inscripción se documenta en otros lugares como el labio interior del vaso, las asas o el pie. En muchos de estos ejemplos cabe ver una tendencia adaptativa de la inscripción al lugar más relevante donde se centra la mirada, a veces en juego, coexistencia o contraste con la decoración figurada: así la conocida inscripción de un tal Sóstrato a la A frodita de Naúcratis está grabada en el interior de un gran cuencio rodio (Museo Británico, 88, 6-1 456), ricamente decorado en ese lugar con frisos de animales orientalizantes y con cabezas femeninas sobre el labio que miran hacia el interior (BOARDMAN, 1973, fig. 139). En un lécito ático de figuras negras del Museo Nacional de Atenas $\left(\mathrm{n}^{\circ}\right.$ 1055) la inscripción corre, pintada, alrededor del pie: un regalo

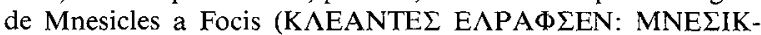
VEI $\triangle E \Sigma:$ EAOKEN: ФOKI)

(7) El fragmento ha sufrido un notable desgaste desde que lo vi, por primera vez, en 1984 . Entonces los trazos de las letras aparecían más precisos (por ejemplo, la misma $\Theta$ ) y se veía entonces con mayor claridad la primera $N$.

(8) JEFFERY, 1976, 242 con bibliografía. Inscripciones del Museo Británico, 1022. Elgin Collection, 1816, 6-10.107. Hemos de señalar, no obstante, la doble lambda en las dedicaciones jonias a Apolo., por ej., GUARDUCCI, 1967, 261, figs. 114-5. 
propio de un personaje masculino, seguramente local, morfologizado dentro de la declinación temática griega, y la $\mathrm{N}$ anterior como la consonante efelcística de la tercera persona en singular de un aoristo.

Esta interpretación es la única posible que sabemos proponer para la lectura del fragmento, pero presenta ante todo el problema del supuesto nombre propio NIETHOS, que no parece ser griego, tanto por una carencia de descripción parlante, habitual en los nombres arcaicos, como por la extraña coloración de su secuencia fonética (9). Nos queda, pues, la opción para interpretarlo como un antropónimo local, pero también en este caso la carencia de datos sobre el polo indígena nos impide cualquier contrastación. Apenas sabemos nada aún sobre antroponimia tartésica, con excepción del seguramente helenizado, e indoeuropeo, nombre parlante de Argantonio, «el de la plata, el floreciente en plata» (10).

El esquema propuesto de la inscripción como ofrenda o regalo a alguien, es normal en las inscripciones de la época y yo diría que seguramente es la fórmula más habitual en los grafitos conservados de la cerámica jonia arcaica, siendo frecuentes estas dedicaciones en los ámbitos sagrados y comerciales. Recordemos las dedicaciones, por ejemplo, a la Afrodita de Naúcratis (11) (especialmente conocidas por los cálices quiotas o naucratitas) 0 , en el mismo mundo jonio, a los dioses Apolo y Hera (cf. nota 4). Basta a veces el dativo con el nombre del dios para indicar de un modo sintético tanto el acto de entrega como el status jurídico de pertenencia del nuevo poseedor: «para Hera», «para Apolo». Otras veces figuran el nombre del donante y el verbo, y, en ocasiones, la motivación de la ofrenda, por ejemplo, «como regalo de amistad» o

(9) Agradecemos en este punto su valiosa información a Prof. P. M. Fraser, Oxford.

(10) Sobre la onomástica tartesia, PALOMAR LAPESA, 1957, 40 y 145: Argantonius de Alconétar. Agradecemos la información sobre este punto al hoy llorado Prof. A. Tovar. Cf., asimismo, ALBERTOS, 1966, 33, 268, 291, 294, 305: Argantonios, Argantonius. Un planteamiento general del problema, ALBERTOS, 1976, 57-86. UNTERMANN, 1957, no recoge ni el nombre ni el problema de Argantonio.

(11) Por ejemplo, sobre el pie de un cáliz quiota en Cam-

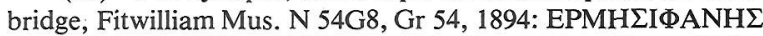
ANE@HKEN THФPO $\triangle I T H$, o el citado cuenco rodio en el Museo Británico (cf. n. 6) hallado en el recinto de la Afrodita de

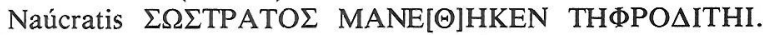
Otros ejemplos, JOHNSTON, 1978.

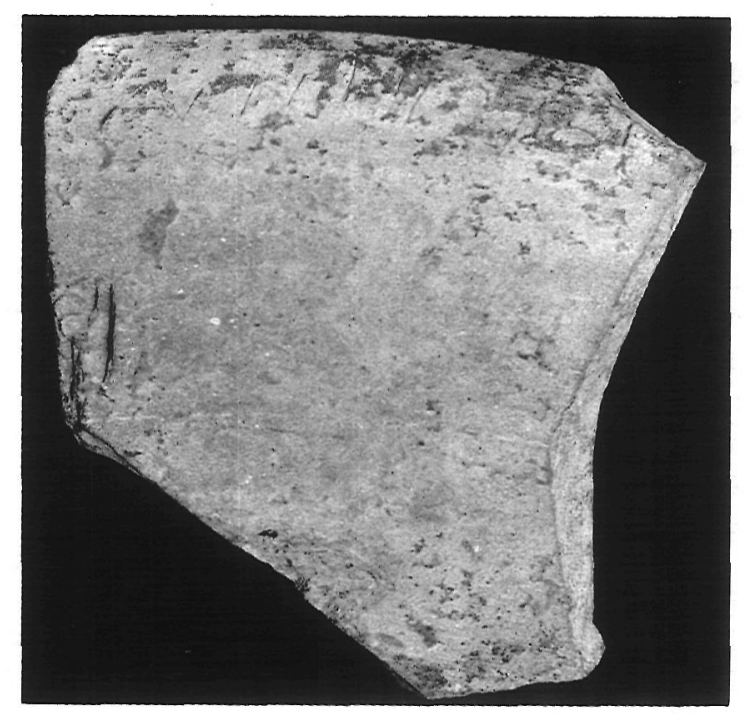

Fig. 3

«como diezmo de los beneficios», tal como se supone figuraría en la perdida inscripción acompañante del gigantesco caldero de bronce ofrendado por Coleo a la diosa Hera de Samos (12).

Esta hipotética propuesta de lectura para el fragmento de Huelva lleva implícito un cúmulo de cuestiones múltiples que, al menos como vía de ensayo lucubrativo, debemos formular: ipor quién, con qué finalidad, dónde, se ha realizado la inscripción? Hoy, sólo es posible contestar a estas cuestiones con hipótesis o con nuevas preguntas.

La presencia de una inscripción jonia arcaica en Huelva sugiere, como un dato más, un complejo mundo de contactos comerciales en el que, de un modo u otro, hubo de estar también presente la escritura - y la ideología y status que ésta implicapara el período que nos ocupa. Tampoco en este aspecto podemos decir que los griegos innoven. Más bien reproducen o continúan una anterior tradición fenicia, del mismo modo que ocurre con el conjunto de la experiencia comercial griega en Tarteso, un emporio abierto en el que unos productos, similares funcionalmente, sustituyen a otros anteriores dentro de un esquema comercial en gran medida ya prefigurado. Con relación a la escritura, probablemente no es otro el impulso que en el Levante español estimulará el origen y desarrollo del

(12) DUNST, 1972, 99: Ensayo reconstructivo. Como expresión o intercambio de amistad, cf. la inscripción sobre el dinos cerámico del Hereo de Samos (inicios del siglo VI) en WALTER-KARYDI, 1973, 9 y nota 21: megàles antì philemosýnes. 
alfabeto grecoibérico, que hoy se documenta en inscripciones de plomo en principio sensiblemente más tardías (DE HOZ 1983, 27-61; DE HOZ, 1984, 351-96).

La escritura, la metrología (el pesaje de la plata que subyace en el texto herodoteo), la actuación divina y el intercambio comercial arcaico con presentes de hospitalidad, son elementos integrantes de la relación entre griegos y tartesios de élite, o incluso de raigambre aristocrática, una aristocracia comercial en crisis por parte griega. Pero, sobre todo, hemos de ver en la escritura un vehículo de realización comercial pragmática.

Dijimos que ha podido realizar esta inscripción un samio o un foceo. No sabemos si se grabó en su lugar de origen o en la misma Tarteso, ni si la realizó el mismo dedicante u otra persona por encargo, con experiencia en la escritura. De ser efectivamente una dedicación a un personaje tartesio con quien el griego traba la relación comercial, ambas posibilidades tendrían su sentido: bien como señal material del recuerdo de quien ya estuvo en el emporio tartesio y envía desde el hogar una tarjeta de identidad, o bien como una especie de testimonio o monimentum realizado en el mismo Port of trade tartesio constancia, por ejemplo, de un pacto de $p h i$ lía comercial así sellado (13).

En la Etruria arcaica conocemos un amplio grupo de dedicaciones, en etrusco, sobre importaciones cerámicas, que son relacionables, como en nuestra conjetural propuesta, con esta práctica del don (CRISTOFANI, 1985). Estas inscripciones son generalmente formulares y en ellas aparecen alternativamente dos verbos relacionados con la acción de ofrendar, sagrada o profana -mulunarike, tureke-, equivalentes, respectivamente, al anétheke y al édoke griegos. Alguien entrega el regalo a alguien, pues en el intercambio de regalos y de pactos es necesario denominar a las dos partes que intervienen. Cristofani $(1975,150$ ss.) interpreta económicamente estas dedicaciones como «relitti di una piú antica economia del dono». ¿Nos hallamos también en nuestro caso aislado ante una situación similar?.

(13) Quiero marginalmente recordar las dedicatorias en pateras de bronce como las mucho más tardías del Norte de España que reseña $\mathrm{DE} \mathrm{HOZ}, 1982,304, \mathrm{n} .^{\circ} 40$ y 42 (con referencias a Untermann, «Namenkundliche Anmerkungen zu lateinischen Inschriften aus Kantabrien» $B N, 15,1980,382$ : una patera utiliza el dativo para la fórmula onomástica. MONIMAN en estas pateras puede relacionarse (J. DE HOZ) con monumentum.
Pero las hipótesis planteables para aproximarnos a una realidad definitivamente desvanecida son en este sentido infinitas y no debemos ver en las aquí ofrecidas sino simples conjeturas de muy difícil o imposible demostración. Pues nos basamos en un fragmento cerámico de lectura especialmente compleja y, hoy por hoy, sin otras posibilidades de contrastación.

\section{BIBLIOGRAFÍA}

ALBERTOS, M. ${ }^{\mathrm{a}}$ L. 1966: La onomástica personal primitiva de Hispania tarraconense y Bética, Salamanca.

ARRIBAS, A. y WILKINS, J. 1971: «La Necrópolis fenicia del Cortijo de las Sombras (Frigiliana, Málaga)», Cuadernos de Prehistoria de la Universidad de Granada, 59.

BOARDMAN, J. 1973: The Greeks Overseas. Londres,

CABRERA, P.y OLMOS, R., 1985: «Die Griechen in Huelva. Zum Stand der Diskussion» en Madrider Mitteilungen, 26, 61-74.

CARRIAZO, J.M. y RADDATZ, K. 1960: «Un corte estratigráfico en Carmona», Archivo Hispalense, 103-152.

CRISTOFANI, M. 1975, «Il 'dono' nell'Etruria arcaica», La Parola del Passato, CLX, 132-152.

DUNST, G. 1972: «Archaische Inschriften und Dokumente der Pentecontaetie aus Samos», Mitteilungen des Deutschen Archäologischen Instituts. Athenische Abteilung, 87, 99149.

FERNANDEZ JURADO, J. 1984: La presencia griega arcaica en Huelva. Excavaciones en Huelva, 1. Huelva.

FERNANDEZ JURADO, J. 1985: «Die Phönizier in Huelva», Madrider Mitteilungen, 26, 49-60.

GUARDUCCI, M. 1964, «Appunti di epigrafia greca arcaica», Archeologia Classica, 16, 122-53.

GUARDUCCI, M. 1967: Epigrafia greca. I. Roma.

HOMANN-WEDEKING, E. 1966: «Ausgrabungen im Heraion von Samos. 1961», Archöologischer Anzeiger, 77-87.

HOMANN-WEDEKING, 1969, ref. en «125 Winckelmannfest 9.12.68», Archäologischer Anzeiger, 554.

DE HOZ, J., 1981: «Las lenguas prerromanas en la Península Ibérica», VI Congreso Español de Estudios Clásicos, Sevilla 1981. Madrid. 351-396.

DE HOZ, J. 1982: «Crónica de Lingüística y Epigrafía de la Península Ibérica: 1981», Zephyrus XXXIV-XXXV, $295-$ 308.

DE HOZ, J. 1983: «Origine ed evoluzzione delle scritture ispaniche). Annuali del Seminario di Studi del Mondo Classico. Sezzione linguistica, 5, 2-61.

JEFFERY, L. H., 1961: The local Scripts of Archaic Greece. Oxford.

JEFFERY, L. H., 1976: Archaic Greece.

JOHNSTON, 1978: Pottery from Naucratis, Londres.

KYRIELEIS, H., 1981: Führer durch das Heraion von Samos. Atenas. 
LUZON, J. M. y RUIZ MATA, D., 1973: «Las raices de Córdoba. Estratigrafía de la Colina de los Quemados». Boletín de la Academia de la Historia de Córdoba.

OLMOS, R. y CABRERA, P., 1980: «Un fragmento de Clitias en Huelva) Archivo Español de Arqueología, 53, 5-14.

OLMOS, R. y GARRIDO, J. P. 1982: «Cerámica griega en Huelva. Un informe preliminar», Homenaje a Sáenz de Buruaga, 243-259.

PALOMAR LAPESA, M., 1957: La onomástica personal prelatina en la antigua Lusitania, Salamanca.

PELLICER, M. y AMORES, F. 1985: «Prehistoria de Carmona», Noticiario Arqueológico Hispánico, 22, 57-189.

PELLICER, M. y otros 1983: «El cerro Macareno». Excavaciones Arqueológicas en España, 124.
PETRIE, W. M. F. 1886: Excavation at Naucratis. Londres.

UNTERMANN, J. 1957: Elemenios de un atlas antroponímico de la Hispania antigua, Madrid.

VOITGLANDER, W, 1982, «Funde aus der Susula Westlich des Bouleuterion in Milet», Istambuler Mitteilungen, 32 , 1982.

WALTER-KARYDI, E, 1973: Samos, VI, I. Bonn.

WILLIAMS, D. 1983: «Aegina. Aphaia Tempel, V. The pottery from Chios» Archäologischer Anzeiger, 1983, 169 178.

WOODHEAD, A. G. 1981: The study of Greek Inscriptions, Cambridge, $2 .^{a}$ ed. 